PROCEEDINGS OF THE AMERICAN MATHEMATICAL SOCIETY

Volume 124, Number 8, August 1996

\title{
ON RELATIVE HAUSDORFF MEASURES OF NONCOMPACTNESS AND RELATIVE CHEBYSHEV RADII IN BANACH SPACES
}

\author{
ANDRZEJ WIŚNICKI AND JACEK WOŚKO \\ (Communicated by Palle E. T. Jorgensen)
}

\begin{abstract}
In this paper we prove some formulae and evaluations on relative Hausdorff measures of noncompactness and relative Chebyshev radii in various Banach spaces. We generalize the Lifschitz constant $\kappa(X)$ and introduce a function $\tilde{\kappa}_{X}(\cdot)$.
\end{abstract}

\section{INTRODUCTION}

In this paper $X$ will always denote a real Banach space and $A$ will be a bounded subset of $X$. The classical Hausdorff measure of noncompactness $\chi(A)$ is defined as the infimum of numbers $\varepsilon>0$ such that $A$ can be covered with a finite number of balls of radii smaller than $\varepsilon$. The absolute Chebyshev radius $r(A)$ is defined as the infimum of numbers $\varepsilon>0$ such that $A$ can be covered with a ball of a radius $\varepsilon$. Thus we have

$$
r(A)=\inf _{y \in X} \sup _{x \in A}\|x-y\| .
$$

The concept of relative Chebyshev centers and radii is a natural generalization of the notion of the absolute Chebyshev center and radius. In particular, relative Chebyshev centers have been well studied in recent years (see for instance [13]). For a given set $\emptyset \neq G \subset X$ the relative radius $r_{G}(A)$ is given by

$$
r_{G}(A)=\inf _{y \in G} \sup _{x \in A}\|x-y\| .
$$

Similarly we can define the relative Hausdorff measure of noncompactness $\chi_{G}(A)$ as the infimum of those $r>0$ such that $A$ can be covered with a finite number of balls with centers in $G$ of radii smaller than $r$. If $G=A$ we have the so-called inner Hausdorff measure of noncompactness. If $G$ is a linear subspace of $X$ and $A \subset G$, we obtain the (classical) Hausdorff measure of noncompactness in a subspace $G$ [16].

Section 2 contains formulae on $\chi_{G}(A)$ and $r_{G}(A)$ in terms of the Hausdorff distance.

Received by the editors September 19, 1994 and, in revised form, February 24, 1995.

1991 Mathematics Subject Classification. Primary 41A65, 46B20, 47H09; Secondary 41A50, $47 \mathrm{H} 10$.

Key words and phrases. Chebyshev radius, Hausdorff measure of noncompactness, Hausdorff distance, Lifschitz constant, $L^{p}$ spaces, space of continuous functions.

(C)1996 American Mathematical Society 
In section 3 we generalize the Lifschitz constant $\kappa(X)$ and give evaluations of $\chi_{G}(A)$ and $r_{G}(A)$ in any Banach spaces.

In section 4 a function $\tilde{\kappa}_{X}(\cdot)$ is defined. With the help of this function we give some stronger evaluations of $\chi_{G}(A)$ and $r_{G}(A)$ than those given in section 3.

Section 5 presents some applications of previous ideas to Hilbert spaces, $L^{p}$ spaces and some spaces with the norm "supremum". We generalize the formula proved by Smith and Ward in [15].

\section{General Remarks}

Let $B(x, r)$ denote the closed ball centered at $x \in X$ with radius $r>0$, and let $\operatorname{dist}(x, A)$ denote the distance to a point $x$ from $A \subset X$. We shall also use the notation:

$$
\begin{gathered}
B(A, r)=\{x \in X: \operatorname{dist}(x, A) \leq r\}, \\
\operatorname{dist}(A, G)=\inf _{x \in A} \inf _{y \in G}\|x-y\|, \quad A, G \subset X .
\end{gathered}
$$

Denote by $\mathcal{M}$ the family of all nonempty bounded subsets of $X$. For $A, G \in \mathcal{M}$ put

$$
\begin{gathered}
d(A, G)=\inf \{r>0: A \subset B(G, r)\}, \\
D(A, G)=\max \{d(A, G), d(G, A)\}
\end{gathered}
$$

and call them the nonsymmetric and symmetric Hausdorff distance between $A$ and $G$, respectively. Sometimes we shall use the symbol $d(A, G)$ with unbounded $G$. It is well known that $D$ is a metric defined on the family of all bounded and closed subsets of $X$. We shall also use the following symbols:

$\mathcal{N}$-the family of all relatively compact and nonempty subsets of $X$,

$\mathcal{N}^{0}$-the family of all nonempty finite subsets of $X$,

$\mathcal{N}^{s}$-the family of all subsets of $X$ consisting of exactly one element.

If $\mathcal{Z}$ is a family of subsets of $X$, then we shall write

$$
d(A, \mathcal{Z})=\inf _{G \in \mathcal{Z}} d(A, G) \text { and } D(A, \mathcal{Z})=\inf _{G \in \mathcal{Z}} D(A, G) .
$$

Using this notation it is easily seen that

$$
\chi(A)=D(A, \mathcal{N})=d(A, \mathcal{N})=D\left(A, \mathcal{N}^{0}\right)=d\left(A, \mathcal{N}^{0}\right)
$$

and

$$
r(A)=D\left(A, \mathcal{N}^{s}\right)=d\left(A, \mathcal{N}^{s}\right) .
$$

Proposition 2.1. Let $\emptyset \neq G \subset X$. Then

$$
\begin{gathered}
\chi_{G}(A)=\inf _{F \in \mathcal{N}^{[}}[d(A, F)+d(F, G)]=\inf _{F \in \mathcal{N}^{0}}[d(A, F)+d(F, G)], \\
r_{G}(A)=\inf _{F \in \mathcal{N}^{s}}[d(A, F)+d(F, G)] .
\end{gathered}
$$

Proof. The equality $\inf _{F \in \mathcal{N}}[d(A, F)+d(F, G)]=\inf _{F \in \mathcal{N}^{0}}[d(A, F)+d(F, G)]$ follows from the fact that for every $\varepsilon>0$ and $F \in \mathcal{N}$ there exists $F_{0} \in \mathcal{N}^{0}$ such that $d\left(F, F_{0}\right)<\varepsilon$ and $d\left(F_{0}, F\right)<\varepsilon$. Therefore it is sufficient to prove that $\chi_{G}(A)=$ $\inf _{F \in \mathcal{N}^{0}}[d(A, F)+d(F, G)]$. Fix $\varepsilon>0$ and $F \in \mathcal{N}^{0}$, and choose $x \in A$. By 
the definition of $d(A, F)$ and $d(F, G)$ there exist $f \in F$ and $g \in G$ such that $\|x-f\| \leq d(A, F)+\frac{\varepsilon}{2}$ and $\|f-g\| \leq d(F, G)+\frac{\varepsilon}{2}$. Hence

$$
\|x-g\| \leq\|x-f\|+\|f-g\| \leq d(A, F)+d(F, G)+\varepsilon
$$

and so

$$
\chi_{G}(A) \leq d(A, F)+d(F, G)
$$

for every $F \in \mathcal{N}^{0}$. This implies

$$
\chi_{G}(A) \leq \inf _{F \in \mathcal{N}^{0}}[d(A, F)+d(F, G)] .
$$

We have

$$
\begin{aligned}
\chi_{G}(A) & \leq \inf _{F \in \mathcal{N}^{0}}[d(A, F)+d(F, G)] \leq \inf _{\substack{F \in \mathcal{N}^{0} \\
F \subset G}}[d(A, F)+d(F, G)] \\
& =\inf _{\substack{F \in \mathcal{N}^{0} \\
F \subset G}} d(A, F)=\chi_{G}(A),
\end{aligned}
$$

which completes the proof. Similar considerations apply to $r_{G}(A)$.

Write

$$
D_{1}(A, B)=d(A, B)+d(B, A) .
$$

Notice that $D_{1}(A, B)$ is a metric defined on the family of all bounded and closed subsets of $X$. From Proposition 2.1 we obtain

Proposition 2.2. Let $A \subset X$ be a bounded, nonempty set. Then

$$
\begin{gathered}
\chi_{A}(A)=D_{1}(A, \mathcal{N})=D_{1}\left(A, \mathcal{N}^{0}\right), \\
r_{A}(A)=D_{1}\left(A, \mathcal{N}^{s}\right) . \quad \square
\end{gathered}
$$

3. Evaluations on $\chi_{G}(A)$ And $r_{G}(A)$ With the uSe OF THE FUNCTION $\kappa_{X}(\cdot)$

For $\varepsilon \geq 0$ write

$$
\begin{gathered}
\mathcal{H}^{\varepsilon}(A)=\left\{F \in \mathcal{N}^{0}: A \subset B(F,(1+\varepsilon) \chi(A))\right\}, \\
E^{\varepsilon}(A)=\{y \in X: A \subset B(y,(1+\varepsilon) r(A))\} .
\end{gathered}
$$

Note that for $\varepsilon>0 \mathcal{H}^{\varepsilon}(A) \neq \emptyset$ and $E^{\varepsilon}(A) \neq \emptyset$ for every bounded $A \subset X$. Proposition 2.1 now gives

Proposition 3.1. Let $\emptyset \neq G \subset X$. Then

$$
\begin{array}{r}
\chi_{G}(A) \leq \chi(A)+\lim _{\varepsilon \rightarrow 0^{+}} d\left(\mathcal{H}^{\varepsilon}(A), G\right), \\
r_{G}(A) \leq r(A)+\lim _{\varepsilon \rightarrow 0^{+}} \operatorname{dist}\left(E^{\varepsilon}(A), G\right) .
\end{array}
$$

To find the converse evaluations on $\chi_{G}(A)$ and $r_{G}(A)$ let us recall the definition of the Lifschitz constant $\kappa(X)$ of a Banach space $X$ :

$$
\begin{aligned}
\kappa(X)=\sup \{k>0 & : \bigvee_{0<\mu, \alpha<1} \bigwedge_{x, y \in X} \bigwedge_{r>0}\|x-y\| \geq(1-\mu) r \\
& \left.\Rightarrow \bigvee_{z \in X} B(x,(1+\mu) r) \cap B(y, k(1+\mu) r) \subset B(z, \alpha r)\right\} .
\end{aligned}
$$


This constant plays an important role in fixed point theorems for uniformly Lipschitzian mappings. We will need to generalize it.

Definition 3.2. Let $X$ be a Banach space. $\kappa_{X}(\cdot)$ is a function defined on $(0,+\infty)$ by

$$
\begin{aligned}
\kappa_{X}(d)=\sup \{k>0 & : \bigvee_{0<\mu, \alpha<1} \bigwedge_{x, y \in X} \bigwedge_{r>0}\|x-y\| \geq(1-\mu) r d \\
& \left.\Rightarrow \bigvee_{z \in X} B(x,(1+\mu) r) \cap B(y, k(1+\mu) r) \subset B(z, \alpha r)\right\} .
\end{aligned}
$$

Theorem 3.3. Let $X$ be a Banach space, $A \subset X$ be a bounded set and $\emptyset \neq G \subset X$. Then

$$
\begin{gathered}
\chi_{G}(A) \geq \chi(A) \kappa_{X}\left(\frac{d_{1}}{\chi(A)}\right) \quad \text { if } \chi(A) \neq 0, \\
r_{G}(A) \geq r(A) \kappa_{X}\left(\frac{d_{2}}{r(A)}\right) \quad \text { if } r(A) \neq 0,
\end{gathered}
$$

where

$$
d_{1}=\lim _{\varepsilon \rightarrow 0^{+}} \sup _{F \in \mathcal{H}^{\varepsilon}(A)} \operatorname{dist}(F, G), \quad d_{2}=\lim _{\varepsilon \rightarrow 0^{+}} d\left(E^{\varepsilon}(A), G\right) .
$$

Proof. Write $\chi_{G}(A)=k, \chi(A)=r$. Assume that $\frac{k}{r}<\kappa_{X}\left(\frac{d_{1}}{r}\right)$. Then we can find $0<\mu, \alpha<1$ such that for every $x, y \in X$ with $\|x-y\| \geq(1-\mu) d_{1}$ there exists $z \in X$ satisfying

$$
B(x,(1+\mu) r) \cap B(y, k(1+\mu)) \subset B(z, \alpha r) .
$$

Choose $g_{1}, g_{2}, \ldots, g_{n} \in G$ satisfying

$$
A \subset \bigcup_{i=1}^{n} B\left(g_{i},(1+\mu) k\right)
$$

and $F=\left\{x_{1}, x_{2}, \ldots, x_{m}\right\} \in \mathcal{H}^{\mu}(A)$ such that

$$
\bigwedge_{\substack{i=1, \ldots, n \\ j=1, \ldots, m}}\left\|g_{i}-x_{j}\right\| \geq \operatorname{dist}(F, G) \geq(1-\mu) d_{1} .
$$

Obviously $A \subset \bigcup_{j=1}^{m} B\left(x_{j},(1+\mu) r\right)$. But

$$
B\left(g_{i},(1+\mu) k\right) \cap B\left(x_{j},(1+\mu) r\right) \subset B\left(z_{i j}, \alpha r\right)
$$

for some $z_{i j} \in X$ and we obtain

$$
\begin{aligned}
A & \subset \bigcup_{i=1}^{n} B\left(g_{i},(1+\mu) k\right) \cap \bigcup_{j=1}^{m} B\left(x_{j},(1+\mu) r\right) \\
& =\bigcup_{i=1}^{n} \bigcup_{j=1}^{m} B\left(g_{i},(1+\mu) k\right) \cap B\left(x_{j},(1+\mu) r\right) \subset \bigcup_{i=1}^{n} \bigcup_{j=1}^{m} B\left(z_{i j}, \alpha r\right),
\end{aligned}
$$

which contradicts $\chi(A)=r$. Thus (2) is proved. The proof for (3) is similar. 
It is now natural to consider: When is $\kappa_{X}(d)>1$ ? First recall that the modulus of convexity of a Banach space $X$ is the function $\delta_{X}:[0,2] \rightarrow[0,1]$ defined by

$$
\delta_{X}(\varepsilon)=\inf \left\{1-\frac{\|x+y\|}{2}:\|x\| \leq 1,\|y\| \leq 1,\|x-y\| \geq \varepsilon\right\}
$$

and

$$
\varepsilon_{0}(X)=\sup \left\{\varepsilon \in[0,2]: \delta_{X}(\varepsilon)=0\right\} .
$$

We follow the ideas of Downing and Turett [3] (see also [8]).

Proposition 3.4. In any Banach space $X$ :

$$
\kappa_{X}(d)>1 \Leftrightarrow \varepsilon_{0}(X)<d .
$$

Proof. Let $\varepsilon_{0}(X)<d$ and let $u \in B(x,(1+\mu) r) \cap B(y,(1+\mu) k r)$, where $\mu>0$, $k>1$ and $\|x-y\| \geq(1-\mu) d r$. Then $\|u-x\| \leq(1+\mu) r,\|u-y\| \leq(1+\mu) r k$ and taking $z=\frac{x+y}{2}$ we obtain

$$
\|u-z\| \leq\left(1-\delta_{X}\left(\frac{(1-\mu) d}{(1+\mu) k}\right)\right)(1+\mu) k r \leq \alpha r
$$

with $\alpha<1$ if $\mu$ is sufficiently small and $k$ is sufficiently close to 1 . Hence $\kappa_{X}(d)>1$ (see also [8]). To prove the converse assume that $\kappa_{X}(d)>1$ and $\varepsilon_{0}(X) \geq d$. Fix $\varepsilon>0$. There exist $x, y \in X$ such that $\|x\|=\|y\|=1,\|x-y\|=d$ and $\left\|\frac{x+y}{2}\right\| \geq 1-\varepsilon$. For any $\mu>0$ the segment

$$
[0, x+y] \subset B(x,(1+\mu)) \cap B(y,(1+\mu)) .
$$

But $\|x+y\| \geq 2-2 \varepsilon$ and $\varepsilon$ is arbitrary so $\kappa_{X}(d)=1$, which is a contradiction.

Notice that for every $0<d<\frac{4}{\varepsilon_{0}(X)}$ there exists exactly one number $b \geq 1$ such that $b\left(1-\delta_{X}\left(\frac{d}{b}\right)\right)=1$ (it follows from the fact that $\delta_{X}$ is an increasing, continuous function on $[0,2)$ and $\left.\lim _{\varepsilon \rightarrow 2^{-}} \delta_{X}(\varepsilon)=1-\frac{\varepsilon_{0}(X)}{2}[7]\right)$. Denote by $b_{X}(\cdot)$ a function depending on the argument $d<\frac{4}{\varepsilon_{0}(X)}$ satisfying

$$
b_{X}(d)\left(1-\delta_{X}\left(\frac{d}{b_{X}(d)}\right)\right)=1 .
$$

Similar considerations to that given above lead us to

Proposition 3.5. In any Banach space $X$ :

$$
\kappa_{X}(d) \geq b_{X}(d) \quad \text { for } d<\frac{4}{\varepsilon_{0}(X)} .
$$

The constant $b_{X}(1)$ was introduced by Goebel and Kirk in [6].

\section{Evaluations on $\chi_{G}(A)$ and $r_{G}(A)$ with the use OF THE FUNCTION $\tilde{\kappa}_{X}(\cdot)$}

In section 3 the expressions $d_{1}, d_{2}$ in Theorem 3.3 are not natural. Now we find stronger and more useful evaluations on $\chi_{G}(A)$ and $r_{G}(A)$. We start with the following definition: 
Definition 4.1. Let $X$ be a Banach space. $\tilde{\kappa}_{X}(\cdot)$ is a function defined on $(0,+\infty)$ by

$$
\begin{array}{r}
\tilde{\kappa}_{X}(d)=\sup \left\{k>0: \bigvee_{0<\alpha<1} \bigwedge_{x, y \in X} \bigwedge_{r>0} \bigvee_{z \in X}\|z-y\| \leq \alpha d r \wedge B(x, r)\right. \\
\cap B(y, k r) \subset B(z, r)\} .
\end{array}
$$

Theorem 4.2. Let $X$ be a Banach space, $A \subset X$ be a bounded set and $\emptyset \neq G \subset X$. Then

$$
\begin{gathered}
\chi_{G}(A) \geq \chi(A) \tilde{\kappa}_{X}\left(\frac{d_{\chi}}{\chi(A)}\right) \quad \text { if } \chi(A) \neq 0, \\
r_{G}(A) \geq r(A) \tilde{\kappa}_{X}\left(\frac{d_{r}}{r(A)}\right) \quad \text { if } r(A) \neq 0,
\end{gathered}
$$

where

$$
d_{\chi}=\lim _{\varepsilon \rightarrow 0^{+}} d\left(\mathcal{H}^{\varepsilon}(A), G\right), \quad d_{r}=\lim _{\varepsilon \rightarrow 0^{+}} \operatorname{dist}\left(E^{\varepsilon}(A), G\right) .
$$

Proof. Write $\chi_{G}(A)=k, \chi(A)=r$. Assume that $\frac{k}{r}<\tilde{\kappa}_{X}\left(\frac{d_{\chi}}{\chi(A)}\right)$. Fix $\varepsilon>0$ and take $0<\delta \leq \varepsilon$ such that $d\left(\mathcal{H}^{\delta}(A), G\right) \geq d_{\chi}(1-\varepsilon)$. Then we can find $\alpha<1$ such that for every $x, y \in X$ there exists $z \in X$ satisfying

$$
\|z-y\| \leq \alpha d_{\chi}(1+\delta) \leq \alpha d_{\chi}(1+\varepsilon)
$$

and

$$
B(x,(1+\delta) r) \cap B(y,(1+\delta) k) \subset B(z,(1+\delta) r) .
$$

Choose $g_{1}, g_{2}, \ldots, g_{n} \in G$ such that

$$
A \subset \bigcup_{i=1}^{n} B\left(g_{i},(1+\delta) k\right)
$$

and $F=\left\{x_{1}, x_{2}, \ldots, x_{m}\right\} \in \mathcal{H}^{\delta}(A)$. Then

$$
B\left(g_{i},(1+\delta) k\right) \cap B\left(x_{j},(1+\delta) r\right) \subset B\left(z_{i j},(1+\delta) r\right)
$$

for some $z_{i j} \in X$ satisfying

$$
\left\|z_{i j}-g_{i}\right\| \leq \alpha d_{\chi}(1+\varepsilon), \quad i=1, \ldots, n, j=1, \ldots, m .
$$

Hence

$$
\begin{aligned}
A & \subset \bigcup_{i=1}^{n} B\left(g_{i},(1+\delta) k\right) \cap \bigcup_{j=1}^{m} B\left(x_{j},(1+\delta) r\right) \\
& =\bigcup_{i=1}^{m} \bigcup_{j=1}^{m} B\left(g_{i},(1+\delta) k\right) \cap B\left(x_{j},(1+\delta) r\right) \subset \bigcup_{i=1}^{n} \bigcup_{j=1}^{m} B\left(z_{i j},(1+\delta) r\right)
\end{aligned}
$$

and $\left\{z_{i j}\right\} \in \mathcal{H}^{\delta}(A)$. From the definition of $d\left(\mathcal{H}^{\delta}(A), G\right)$ there exist $i_{0}, j_{0}$ such that

$$
\left\|z_{i_{0} j_{0}}-g_{i_{0}}\right\| \geq d\left(\mathcal{H}^{\delta}(A), G\right) \geq d_{\chi}(1-\varepsilon),
$$

which contradicts (6) if $\varepsilon$ is sufficiently small. Thus (4) is proved. The proof for (5) is similar. 
Let $l_{x, y}$ denote the line $\{\alpha x+\beta y: \alpha, \beta \in \mathcal{R}, \alpha+\beta=1\}$ containing $x, y \in X$, and let $[x, y]$ denote the segment $\{\alpha x+\beta y: \alpha, \beta \geq 0, \alpha+\beta=1\}$. In the next section we will need the following

Lemma 4.3. In any Banach space $X$ and for every $d>0$ we have

$$
\tilde{\kappa}_{X}(d) \geq \tilde{\kappa}_{X}^{l i n}(d)
$$

where

$$
\begin{aligned}
\tilde{\kappa}_{X}^{l i n}(d)=\sup & \left\{k>0: \bigvee_{0<\alpha<1} \bigwedge_{x, y \in X} \bigwedge_{r>0}\|x-y\| \leq r d\right. \\
& \left.\Rightarrow \bigvee_{z \in l_{x, y}}\|z-y\| \leq \alpha r d \wedge B(x, r) \cap B(y, k r) \subset B(z, r)\right\} .
\end{aligned}
$$

Proof. Obviously $d-1 \leq \tilde{\kappa}_{X}^{\text {lin }}(d) \leq d+1$. It is sufficient to consider $\tilde{\kappa}_{X}^{\text {lin }}(d)>d-1$. Assume that $\tilde{\kappa}_{X}(d)<\tilde{\kappa}_{X}^{\operatorname{lin}}(d)$ for some $d>0$. Then there exist $\alpha<1, x, y \in X$, $r>0$ satisfying $\|x-y\|>d r$ and $x_{1}, z \in[x, y]$ such that $\|z-y\| \leq \alpha r d,\left\|x_{1}-y\right\|=d r$ and

$$
B(x, r) \cap B(y, k r) \not \subset B(z, r) \supset B\left(x_{1}, r\right) \cap B(y, k r) .
$$

That means there exists $g \in X$ satisfying $\|g-x\|<r,\|g-y\| \leq k r$ and $\left\|g-x_{1}\right\|>r$. Let $y_{1} \in l_{x, y}$ and $\left\|y-y_{1}\right\|=k r$. Then $\left\|x_{1}-y_{1}\right\|<r$ and we have $\left\|x_{1}-g_{1}\right\|=r$ for some $g_{1} \in\left[y_{1}, g\right]$. Moreover $\left\|g_{1}-y\right\| \leq k r$ and thus $\left\|g_{1}-z\right\| \leq r$. But $\left\|g_{1}-x\right\|<r$ (since $\left\|x-y_{1}\right\| \leq r$ and $\|x-g\|<r$ ). This contradicts $\left\|x_{1}-g_{1}\right\|=r$.

\section{Applications}

In this section we use the results of section 4 . First observe that (in particular) in reflexive spaces $E^{0}(A) \neq \emptyset$ for every bounded set $A$ (see [5], [9] for more details).

Let $H$ be a Hilbert space. It is not difficult to verify that $\kappa_{H}(d)=\sqrt{1+d^{2}}$ and $\tilde{\kappa}_{H}(d)=\sqrt{1+d^{2}}$. Combining Proposition 3.1 and Theorem 4.2 we get

Corollary 5.1. Let $H$ be a Hilbert space, $A \subset H$ be a bounded set and $\emptyset \neq G \subset H$. Then

$$
\begin{aligned}
& \lim _{\varepsilon \rightarrow 0^{+}} \sqrt{[\chi(A)]^{2}+\left[d\left(\mathcal{H}^{\varepsilon}(A), G\right)\right]^{2}} \leq \chi_{G}(A) \leq \chi(A)+\lim _{\varepsilon \rightarrow 0^{+}} d\left(\mathcal{H}^{\varepsilon}(A), G\right), \\
& \sqrt{[r(A)]^{2}+\left[\operatorname{dist}\left(E^{0}(A), G\right)\right]^{2}} \leq r_{G}(A) \leq r(A)+\operatorname{dist}\left(E^{0}(A), G\right) .
\end{aligned}
$$

Let $X=L^{p}$ or $l^{p}, p>2$. We will adapt methods contained in [11], where it is proved that

$$
\|t x+(1-t) y\|^{p}+t^{\frac{p}{2}}(1-t)^{\frac{p}{2}}\|x-y\|^{p} \leq t\|x\|^{p}+(1-t)\|y\|^{p}
$$

for $x, y \in X, 0 \leq t \leq 1$. Take $\|x\| \leq r(1+\mu),\|y\| \leq r k(1+\mu)$ and $\|x-y\| \geq(1-\mu) d r$. We have

$$
\|t x+(1-t) y\| \leq\left(t(1+\mu)^{p}+(1-t)(1+\mu)^{p} k^{p}-t^{\frac{p}{2}}(1-t)^{\frac{p}{2}}(1-\mu)^{p} d^{p}\right)^{\frac{1}{p}} r .
$$

If we find $t_{0} \in(0,1)$ such that

$$
t_{0}+\left(1-t_{0}\right) k^{p}-t_{0}^{\frac{p}{2}}\left(1-t_{0}\right)^{\frac{p}{2}} d^{p}<1,
$$


then $k<\kappa_{X}(d)$. Moreover $\left\|t_{0} x+\left(1-t_{0}\right) y-y\right\|=t_{0}\|x-y\| \leq t_{0} d r$ if $\|x-y\| \leq d r$. Hence there would be also $k<\tilde{\kappa}_{X}^{\text {lin }}(d)$. But (7) is equivalent to

$$
k<\left(1+t_{0}^{\frac{p}{2}}\left(1-t_{0}\right)^{\frac{p}{2}-1} d^{p}\right)^{\frac{1}{p}} .
$$

Putting $t_{0}=\frac{p}{2(p-1)}$ we have $k<\left(1+\frac{d^{p}}{2^{p-1}} p^{\frac{p}{2}}(p-1)^{1-p}(p-2)^{\frac{p}{2}-1}\right)^{\frac{1}{p}}$. Hence (see also [11])

$$
\kappa_{X}(d) \geq\left(1+\frac{d^{p}}{2^{p-1}} p^{\frac{p}{2}}(p-1)^{1-p}(p-2)^{\frac{p}{2}-1}\right)^{\frac{1}{p}}>\left(1+\frac{d^{p}}{2^{p-1}}\right)^{\frac{1}{p}}
$$

and

$$
\tilde{\kappa}_{X}^{l i n}(d) \geq\left(1+\frac{d^{p}}{2^{p-1}} p^{\frac{p}{2}}(p-1)^{1-p}(p-2)^{\frac{p}{2}-1}\right)^{\frac{1}{p}}>\left(1+\frac{d^{p}}{2^{p-1}}\right)^{\frac{1}{p}} .
$$

Proposition 3.1, Theorem 4.2 and Lemma 4.3 yield

Corollary 5.2. Let $X=L^{p}$ or $l^{p}, p>2, A \subset X$ be a bounded set and $\emptyset \neq G \subset X$. Then

$$
\begin{aligned}
& \lim _{\varepsilon \rightarrow 0^{+}}\left([\chi(A)]^{p}+\frac{\left[d\left(\mathcal{H}^{\varepsilon}(A), G\right)\right]^{p}}{2^{p-1}} p^{\frac{p}{2}}(p-1)^{1-p}(p-2)^{\frac{p}{2}-1}\right)^{\frac{1}{p}} \\
& \quad \leq \chi G(A) \leq \chi(A)+\lim _{\varepsilon \rightarrow 0^{+}} d\left(\mathcal{H}^{\varepsilon}(A), G\right), \\
& \left([r(A)]^{p}+\frac{\left[\operatorname{dist}\left(E^{0}(A), G\right)\right]^{p}}{2^{p-1}} p^{\frac{p}{2}}(p-1)^{1-p}(p-2)^{\frac{p}{2}-1}\right)^{\frac{1}{p}} \\
& \quad \leq r_{G}(A) \leq r(A)+\operatorname{dist}\left(E^{0}(A), G\right) .
\end{aligned}
$$

Let $X=L^{p}$ or $l^{p}, 1<p \leq 2$. Lim, $\mathrm{Xu}$, and $\mathrm{Xu}$ [12] and Smarzewski [14] proved that

$$
\|t x+(1-t) y\|^{2}+(p-1) t(1-t)\|x-y\|^{2} \leq t\|x\|^{2}+(1-t)\|y\|^{2}
$$

for $x, y \in X$ and $0<t<1$. Similar considerations to those given above give $\kappa_{X}(d) \geq \sqrt{1+(p-1) d^{2}}$ and $\tilde{\kappa}_{X}^{\text {lin }}(d) \geq \sqrt{1+(p-1) d^{2}}$. Hence we get

Corollary 5.3. Let $X=L^{p}$ or $l^{p}, 1<p \leq 2, A \subset X$ be a bounded set and $\emptyset \neq G \subset X$. Then

$$
\begin{gathered}
\lim _{\varepsilon \rightarrow 0^{+}} \sqrt{[\chi(A)]^{2}+(p-1)\left[d\left(\mathcal{H}^{\varepsilon}(A), G\right)\right]^{2}} \leq \chi_{G}(A) \leq \chi(A)+\lim _{\varepsilon \rightarrow 0^{+}} d\left(\mathcal{H}^{\varepsilon}(A), G\right), \\
\sqrt{[r(A)]^{2}+(p-1)\left[\operatorname{dist}\left(E^{0}(A), G\right)\right]^{2}} \leq r_{G}(A) \leq r(A)+\operatorname{dist}\left(E^{0}(A), G\right) .
\end{gathered}
$$

Let $X=C([0,1], \mathcal{R})$ be the space of real, continuous functions defined on $[0,1]$. We have $\kappa_{X}(d)=\max \{1, d-1\}$ so Theorem 3.3 is not valid in this case. However we may use the function $\tilde{\kappa}_{X}(\cdot)$. We formulate our theorem in a more general case:

Theorem 5.4. Let $M(T, \mathcal{R})$ be the space of bounded, real functions defined on $T$ with the norm $\|x\|=\sup _{t \in T}|x(t)|$. Let $X$ be a linear subspace of $M(T, \mathcal{R})$ containing the function $x(t) \equiv 1$ and closed with respect to taking maximum and minimum, i.e.:

$$
\bigwedge_{x, y \in X} z(t)=\max \{x(t), y(t)\} \in X, \quad \bigwedge_{x, y \in X} z(t)=\min \{x(t), y(t)\} \in X .
$$


If $A \subset X$ is bounded and $\emptyset \neq G \subset X$, then

$$
\begin{gathered}
\chi_{G}(A)=\chi(A)+\lim _{\varepsilon \rightarrow 0^{+}} d\left(\mathcal{H}^{\varepsilon}(A), G\right), \\
r_{G}(A)=r(A)+\lim _{\varepsilon \rightarrow 0^{+}} \operatorname{dist}\left(E^{\varepsilon}(A), G\right) .
\end{gathered}
$$

Proof. It is enough to show that $\tilde{\kappa}_{X}(d)=1+d$. Let $x, y \in X, 0<\alpha<1, r>0$, $k<1+d$ and $u \in B(x, r) \cap B(y, k r)$. Write:

$$
z(t)= \begin{cases}y(t)+(k-1) r & \text { if } x(t) \geq y(t)+(k-1) r, \\ x(t) & \text { if } y(t)-(k-1) r<x(t)<y(t)+(k-1) r, \\ y(t)-(k-1) r & \text { if } x(t) \leq y(t)-(k-1) r .\end{cases}
$$

Then $\|z-y\| \leq(k-1) r \leq \alpha d r$ if $\alpha$ is sufficiently close to 1 . Moreover $\|u-z\| \leq r$ so $u \in B(z, r)$ and thus $\tilde{\kappa}_{X}(d) \geq 1+d$. The inequality $\tilde{\kappa}_{X}(d) \leq 1+d$ is obvious.

Remark 5.5. In particular Theorem 5.4 is valid for $C([0,1], \mathcal{R}), c, c_{0}, l^{\infty}$.

Remark 5.6. The formula (9) in the case of continuous functions was first proved by Smith and Ward in [15] and then by Franchetti and Cheney in [4].

Remark 5.7. The case $G=\left\{x \in X: \int_{[0,1]} x d \mu=0\right\}$ if $X=C([0,1], \mathcal{R})$, where $\mu$ is a real, normalized Borel measure on $[0,1]$, was studied in [16]:

$$
\chi_{G}(A)=\max \left\{\chi(A), \omega^{+}(A), \omega^{-}(A)\right\},
$$

where

$$
\begin{gathered}
\chi(A)=\frac{1}{2} \lim _{h \rightarrow 0^{+}} \sup _{x \in A} \sup \{|x(t)-x(s)|:|t-s| \leq h, t, s \in[0,1]\}, \\
\omega^{+}(A)=\lim _{h \rightarrow 0^{+}} \sup _{x \in A}\left(\int_{[0,1]} R_{h}^{+} x d \mu_{1}-\int_{[0,1]} R_{h}^{-} x d \mu_{2}\right), \\
\omega^{-}(A)=\lim _{h \rightarrow 0^{+}} \sup _{x \in A}\left(\int_{[0,1]} R_{h}^{+} x d \mu_{2}-\int_{[0,1]} R_{h}^{-} x d \mu_{1}\right), \\
\left(R_{h}^{+} x\right)(t)=\sup \{x(t+\theta h):-1 \leq \theta \leq 1, t+\theta h \in[0,1]\}, \\
\left(R_{h}^{-} x\right)(t)=\inf \{x(t+\theta h):-1 \leq \theta \leq 1, t+\theta h \in[0,1]\},
\end{gathered}
$$

$\mu=\mu_{1}-\mu_{2}$ is the Jordan decomposition of $\mu$.

\section{REFERENCES}

1. P. P. Akhmerov, M. J. Kamenskiı̌, A. S. Potapov et al., Measures of noncompactness and condensing operators, Nauka, Novosybirsk, 1986. (Russian) MR 88f: 47048

2. J. Banaś and K. Goebel, Measures of noncompactness in Banach spaces, Lecture Notes in Pure and Applied Math., New York and Basel, 1980. MR 82f:47066

3. D. J. Downing and B. Turett, Some properties of the characteristic of convexity relating to fixed point theory, Pacific J. Math. 104 (1983), 343-350. MR 84b:47070

4. C. Franchetti and E. W. Cheney, Simultaneous approximation and restricted Chebyshev centers in function spaces, in Approximation Theory and Applications, Academic Press, New York, 1981, pp. 65-88. MR 82f:41042

5. A. Garkavi, The best possible net and the best possible cross-section of a set in a normed space, Izv. Akad. Nauk SSSR 26 (1962), 87-106. (Russian) (Translated in Amer. Math. Soc. Transl., Ser. 2, 39 (1964).) MR 25:429 
6. K. Goebel and W. A. Kirk, A fixed point theorem for transformations whose iterates have uniform Lipschitz constant, Studia Math. 67 (1973), 135-140. MR 49:1242

7. _ Topics in metric fixed point theory, Cambridge Univ. Press, London, 1990. MR 92c: 47070

8. K. Goebel and S. Reich, Uniformly convexity, nonexpansive mappings, hyperbolic geometry, Marcel Dekker, New York, 1984. MR 86d:58012

9. R. B. Holmes, A course on optimization and best approximation, Lecture Notes in Math., vol. 257, Springer-Verlag, New York, 1972. MR 54:8381

10. E. A. Lifschitz, Fixed point theorems for operators in strongly convex spaces, Voronež Gos. Univ. Trudy Mat. Fak. 16 (1975), 23-28. (Russian)

11. T. C. Lim, Fixed point theorems for uniformly Lipschitzian mappings in $L^{p}$ spaces, Nonlinear Anal. TMA 7 (1983), 555-563. MR 84g:47050

12. T. C. Lim, H. K. Xu, and Z. B. Xu, Some $L^{p}$ inequalities and their applications to fixed point theory and approximation theory, in Progress in Approximation Theory, Academic Press, New York, 1991, pp. 609-624. MR 92j:47112

13. I. Singer, Best approximation in normed linear spaces by elements of linear subspaces, Springer-Verlag, New York and Berlin, 1970. MR 42:4937

14. R. Smarzewski, On an inequality of Bynum and Drew, J. Math. Anal. Appl. 150 (1990), 146-150. MR 91g:47047

15. P. W. Smith and I. D. Ward, Restricted centers in $C(\Omega)$, Proc. Amer. Math. Soc. 48 (1975), 165-172. MR 52:1127

16. A. Wiśnicki, Hausdorff measure of noncompactness in subspaces of continuous functions of codimension one, Nonlinear Anal. 25 (1995), 223-228. CMP 95:14

Department of Mathematics, UMCS, Pl. M. C. Skeodowskiej 1, 20-031 Lublin, Poland

E-mail address: awisnic@golem.umcs.lublin.pl

E-mail address: jwosko@golem.umcs.lublin.pl 